\title{
A New Laser Beam Pointing Method Using Laser Arrays
}

\author{
P. M. Goorjian* \\ NASA Ames Research Center, M. S. 258-1, Moffett Field, CA 94035-1000
}

\begin{abstract}
A new method is described for optical data transmissions from satellites using laser arrays for laser beam pointing. It combines a lens system and a vertical-cavity surface-emitting laser (VCSEL)/Photodetector Array, both mature technologies, in a novel way. This system is applied to satellites in low-Earth orbit, (LEO). It can replace current architectures which use dynamical systems, (i.e., moving parts) to point the laser, and which may use vibration isolation platforms. Results of computer simulations show diffraction limited beam propagation. Possible additional applications are to planetary distances (deep space optical communications, (DSOC)), to optical multiple access, (OMA), to communication between a constellation of close satellites, and to satellites that use modulating retro-reflectors.

Keywords: laser beam pointing, free-space optical communication, low-Earth orbit (LEO) data communications, integrated VCSEL/ photodetector arrays, deep space optical communications (DSOC), optical multiple access (OMA), constellation of close satellites, modulating retro-reflectors
\end{abstract}

\section{INTRODUCTION}

A new method $\frac{1-3}{3}$ is described for optical data transmissions from satellites using laser arrays for laser beam pointing. The system is simple, static, and compact, and provides accurate pointing, acquisition, and tracking (PAT). It combines a lens system and a VCSEL/Photodetector Array, both mature technologies, in a novel way for PAT. It can improve the PAT system's size, weight, and power (SWaP) in comparison to current systems. Preliminary analysis $\underline{\underline{2}}$ indicates that this system can be incorporated into a small telescope to make the steering finer and consequently be applicable to transmissions between satellites in low-Earth orbit (LEO) and ground terminals. Laser beam pointing is very challenging for LEO, including science missions ${ }^{4}$. Current architectures use dynamical systems, (i.e. moving parts), (fast-steering mirrors (FSM), and/or gimbals) to turn the laser to point to the ground terminal, and some use vibration isolation platforms as well. These dynamical systems could be replaced with the static system described here. Results of computer simulations will be shown, including results for a wide-angle lens $\left(28^{\circ}\right.$ field) that shows diffraction-limited beam propagation. For these electro-optical systems, reaction times to pointing changes and vibrations are on the nanosecond time scale, much faster than those for mechanical systems. For LEO terminals, slew rates are not a concern with this new system. Possible additional applications are to planetary distances (deep space optical communications, DSOC), to optical multiple access (OMA), since laser beams from different sources come in at different angles and then get mapped to different photodetectors; as a result, different beams can be sent back to different sources, and to communication among a constellation of close satellites, which is similar to OMA.

Also, this system is applicable to satellites that use modulating retro-reflectors ${ }^{5}$ by increasing the returning beam power. Current planned architectures envision a high-powered laser at the ground terminal and a modulating retro-reflector at the satellite. The retro-reflector can be replaced with a VCSEL array in the satellite for a strong return response to a weak signal laser beam. This replacement allows for the use of low power lasers at the ground terminal. Also, backscatter, often a limiting factor with a retro-reflected beam, is reduced by using a weaker laser beam from the ground and returning a strong response signal from the satellite.

Section 2 will describe the new concept including background information on VCSEL / Photodetector Arrays and will present preliminary computer simulations using the optics code, OpticStudio, from Zemax, LLC ${ }^{-}$. These simulations use the capabilities in the Physical Optics Propagation option in the code to model the laser source and diffraction effects

* peter.m.goorjian@nasa.gov (650) 604-5547 
from wave optics. These capabilities make it possible to model laser beam propagation over long space communication distances. Section 3 will present results for a wide-angle lens ${ }^{6-7}$, Double Gauss $28^{\circ}$ field, which show diffraction limited beam propagation. The lens data were available in the code OpticStudio and this type of lens is available commercially ${ }^{?}$. Section 4 will discuss the application of this system to LEO. Section 5 will present related topics. Section 6 will present a brief summary of these developments.

\section{NEW CONCEPT FOR LASER BEAM POINTING}

\subsection{Technical Approach}

As Fig. 1 shows, a signal laser beam (green or blue, with rightward arrows), transmitted from a ground terminal, enters the lens system, which directs it to an element of the pixel array (gray rectangle). Each element, or pixel, consists of a VCSEL component/Photodetector pair. The photodetector detects the possibly weak signal beam, and the VCSEL component returns a strong modulated beam to the lens system (green or blue, with leftward arrows), which sends it to the ground terminal. As the signal beam changes direction, e.g., from the blue to the green incoming direction, this change is detected by the photodetectors, and a laser adjacent to the detecting photodetector is turned on to keep the outgoing laser beam on target. Alternatively, a VCSEL component from a different element could send the returning beam along a slightly different outgoing path to account for "pointing ahead". The laser beams are incoherent to avoid interference effects. With incoherent outgoing beams from adjacent lasers, they can overlap so that the returning beam continues to point at the ground terminal without self-interference.

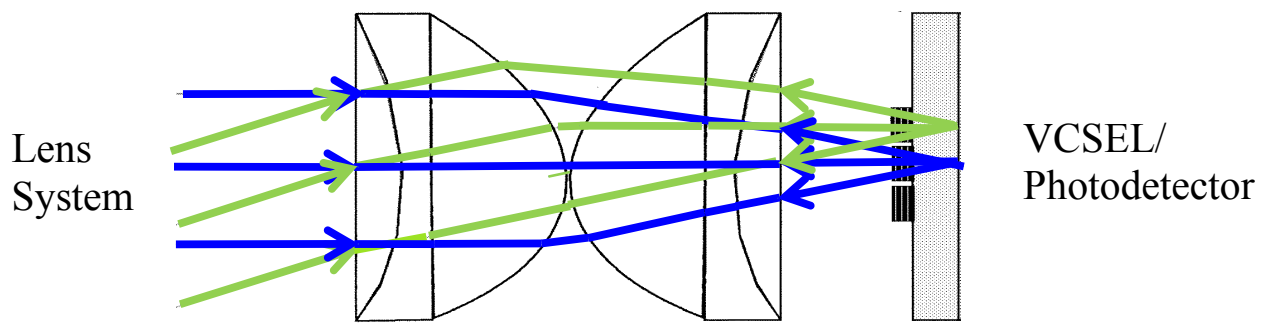

Fig. 1: Space Optical Communications using a Lens System with a Vertical Cavity Surface Emitting Laser (VCSEL)/ Photodetector Array

The outgoing beam can be much stronger than the incoming signal beam, depending only on the power of the VCSEL. For various levels of outgoing power, the VCSEL component may consist of a single VCSEL or a cluster of VCSELs. The use of a cluster of VCSELs rather than a larger aperture VCSEL is to maintain the restriction to a single-mode laser beam. The VCSEL cluster will emit several incoherent beams that are all single-mode. This restriction is desirable to facilitate the detection of the beam at the receiver by avoiding multi-modes in the beam.

\subsection{VCSEL/ Photodetector Array}

Figure 2 shows a candidate VCSEL/ Photodetector Array. In this array the pitch, (i.e. the distance between elements), is the same for the VCSEL clusters and the photodetectors. Also, this packing pattern makes the distance between adjacent elements equal.

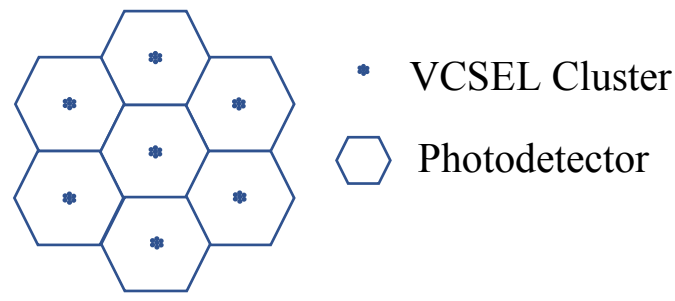

Fig. 2: Candidate VCSEL/ Photodetector Array 
Figure 3 shows an example ${ }^{8}$ of a fabricated VCSEL/ Photodetector Array. The Photodetectors are p-type/intrinsic/ntype (PIN) detectors.

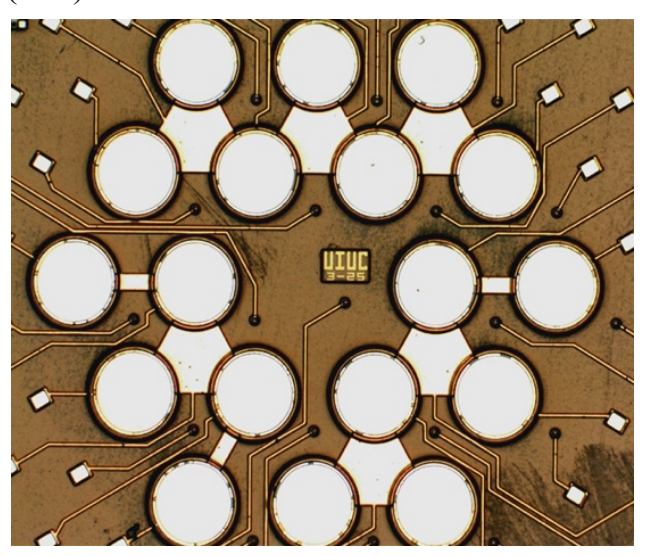

3a)

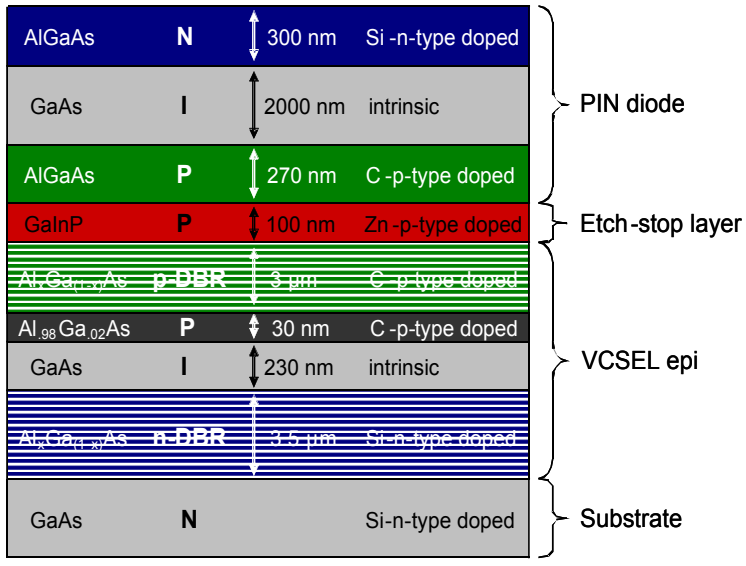

3b)

Figure 3: a) Top-view of a 37-element VCSEL / PIN detector array. VCSELs are small black dots and photodetectors are large white circles, b) Side-view of the epi-structure used for the fabrication of integrated VCSEL / PIN detector arrays.

\subsection{Preliminary Simulations}

Figure 4 shows the wave front of 19 laser beams at propagation distances of $1 \mathrm{~km}$ and $500 \mathrm{~km}$. The integrity of each beam is maintained as it propagates from the lens system. The spot size of the wave front of the cluster and each of the individual laser beams is dependent on the propagation distance and the lens system. In addition, the spot size of the wave front of the cluster is dependent on the array parameters, such as the pitch, (i.e. distance between the centers of the individual lasers), and the spot size of the individual laser beams depends on the laser parameters, such as the aperture diameter of the individual lasers. The wavelength of the beam is $850 \mathrm{~nm}$, which is a wavelength for which VCSELs are well developed for applications.
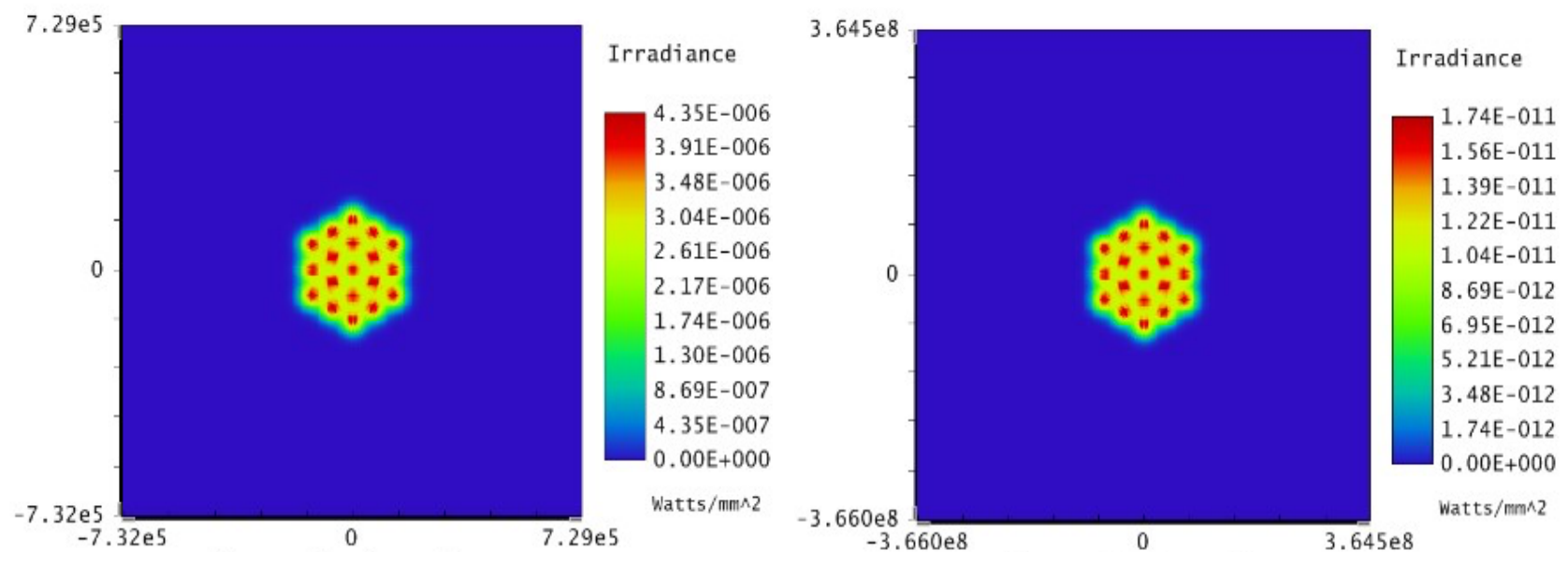

Figure 4: The wave front of 19 laser beams at propagation distances of $1 \mathrm{~km}$ and $500 \mathrm{~km}$. The unit of distance in the plane of the wave front is millimeters.

Figure 5 shows $^{\underline{7}}$ propagation through a relay ${ }^{\underline{6}}$ lens system (diffraction limited) of two overlapping laser beams. As the direction of a target moves away from an active laser beam, as detected by the photodetectors, an adjacent laser is turned 
on to keep the laser beam on target and the first laser is turned off. Alternately, multiple, e.g., three, lasers could be turned on to provide a peak intensity plateau.

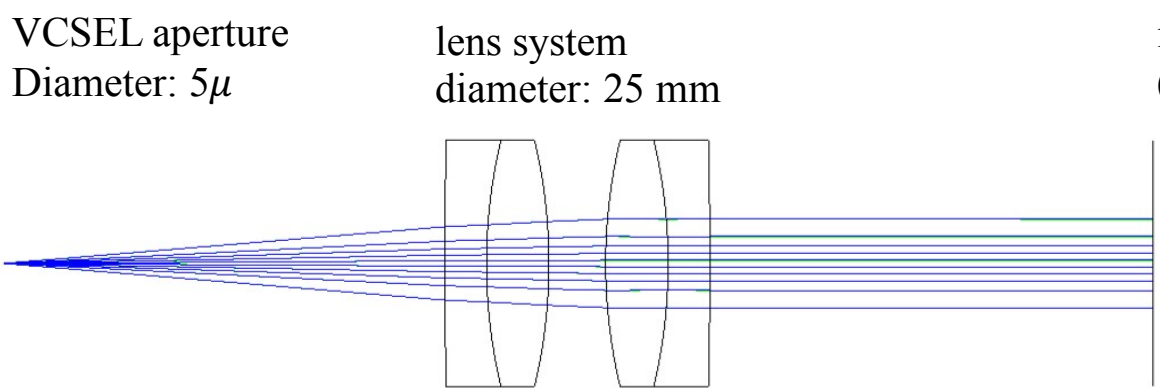

Figure 5: Propagation of two overlapping laser beams.

\author{
irradiance at LEO \\ $(500 \mathrm{~km})$; spot size $1 \mathrm{~km}$
}

\section{A Wide-Angle Lens, Double Gauss $28^{\circ}$ Field}

Computer simulation ${ }^{7}$ results are shown below for a wide-angle lens ${ }^{\frac{6-7}{}}$, which shows diffraction-limited beam propagation to a LEO distance of $611 \mathrm{~km}$. The angular field of propagation is $28^{\circ}$, which shows that this method of pointing a laser beam is not limited to small angles, as long as the lens system is designed adequately. The results show the irradiance and phase for outgoing beams pointed at several angles from $0^{\circ}$ to $14^{\circ}$. These beams are diffraction limited. Also, results for incoming beams are shown.

Figure 6 shows the lens system ${ }^{7}$ and the focusing at the focal plane of four beams (colors) that are incoming from the left side at different angles, including $0^{\circ}$ (blue) and $14^{\circ}$ (red). Each beam enters as colinear.

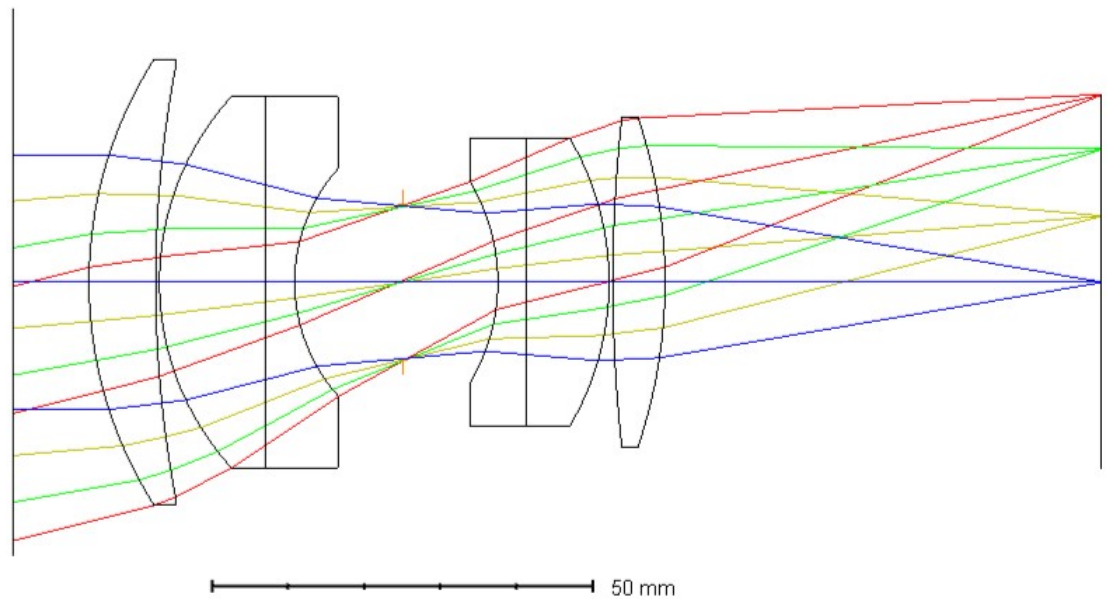

Figure 6: Double Gauss $28^{\circ}$ Field Lens system and incoming colinear beams.

Figure 7 shows laser beams being emitted from lasers at the focal plane and propagating out of the lens system at several angles, including those at $0^{\circ}$ and $14^{\circ}$. Each beam leaves as colinear. These outgoing beams have a wavelength of 850 $\mathrm{nm}$. They are incoherent to avoid interference effects. 


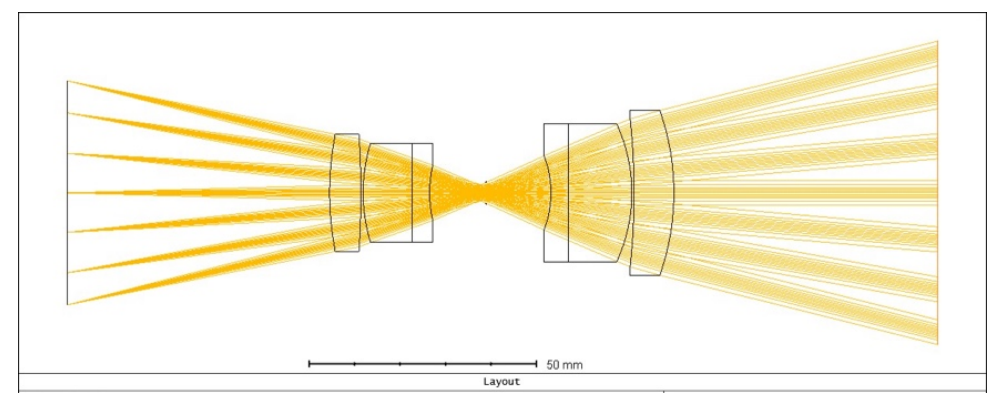

Figure 7: Double Gauss $28^{\circ}$ Field Lens system and outgoing colinear laser beams.

\subsection{Irradiance and Phase for Outgoing Laser Beams}

Figure 8 shows the irradiance and phase of the wave front at $611 \mathrm{~km}$ for a laser beam that is pointed at $0^{\circ}$ and has $22 \mathrm{~mW}$ of power. The irradiance has a Gaussian profile, and the phase is essentially flat, so that this beam is diffraction limited. The phase is shown for irradiance above $0.1 \%$.
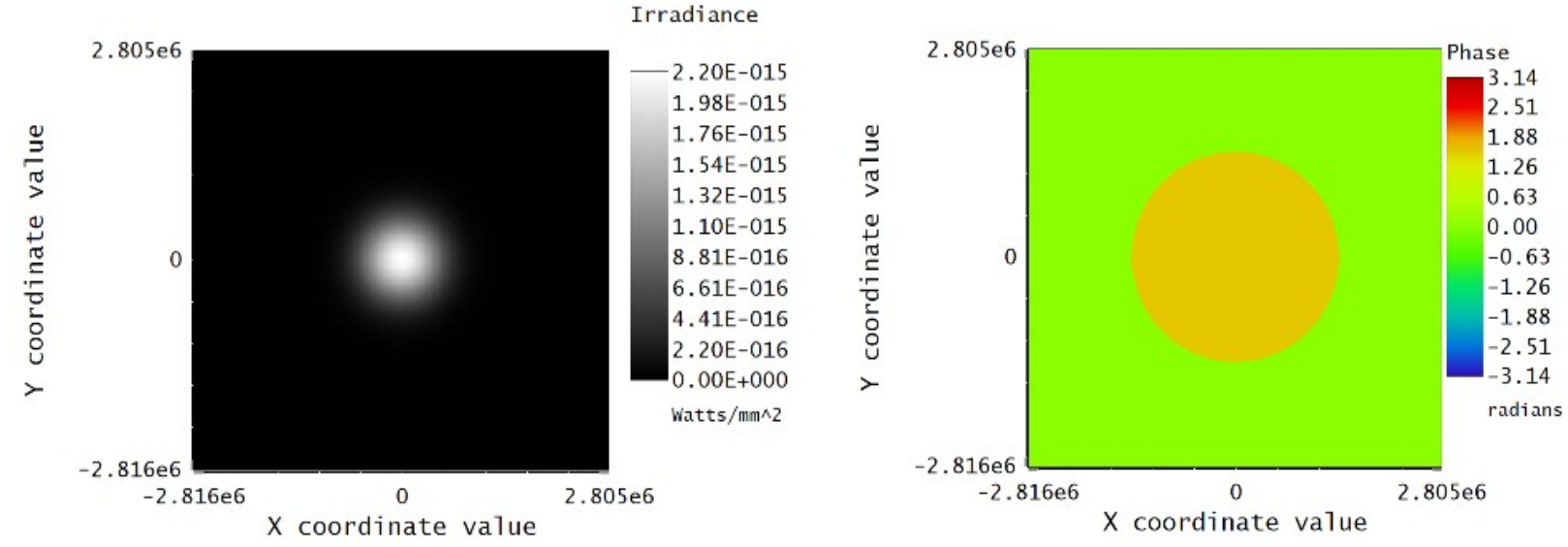

Figure 8: A laser beam that is pointed at $0^{\circ}$ with $2 \mathrm{~mW}$ of power. Coordinates units are in $\mathrm{mm}$.

Figure 9 shows the irradiance and phase of the wave front at $611 \mathrm{~km}$ for a laser beam that is pointed at $14^{\circ}$ and has 2 $\mathrm{mW}$ of power. The irradiance has a Gaussian profile, and the phase is essentially flat, so that this beam is diffraction limited.
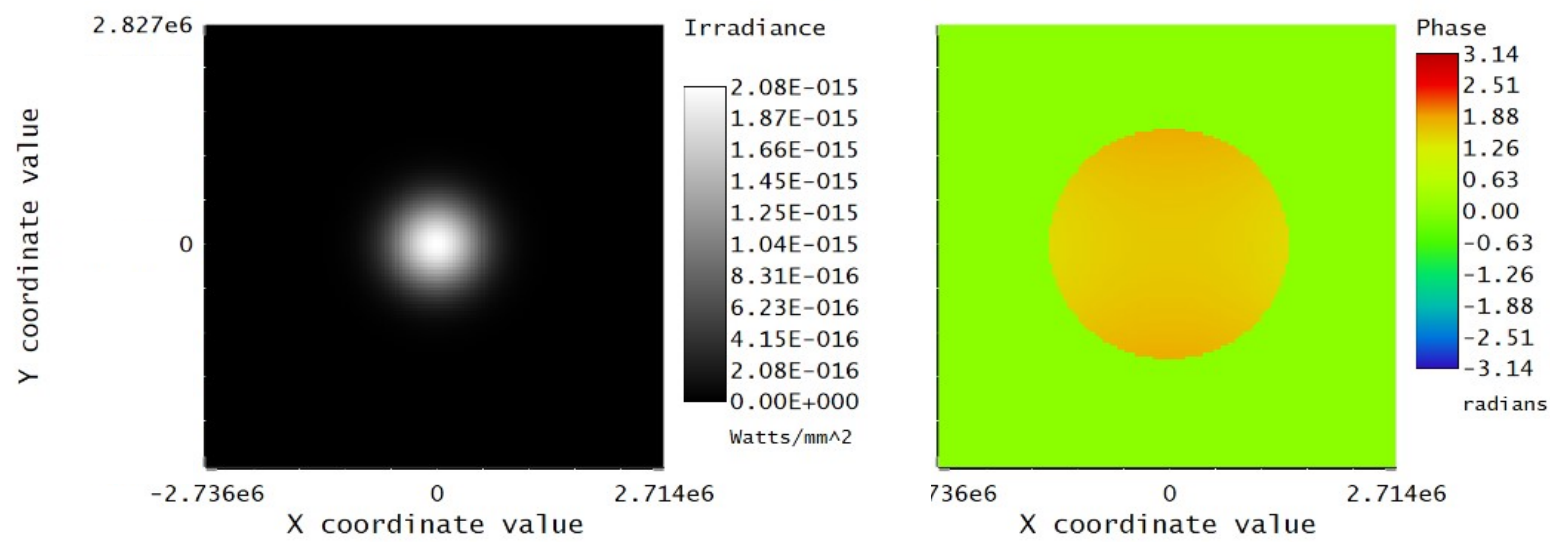

Figure 9: A laser beam that is pointed at $14^{\circ}$ with $2 \mathrm{~mW}$ of power. Coordinates values are $\mathrm{mm}$. 
To account for the signal beam changing direction, a second laser needs to be turned such that the two beams overlap. Figure 10 shows the irradiance at $611 \mathrm{~km}$ for two overlapping incoherent laser beams that are pointed at $0^{\circ}$ and $0.075^{\circ}$ and which both have $2 \mathrm{~mW}$ of power. The phase of the beam pointed at $0.075^{\circ}$ is also shown.

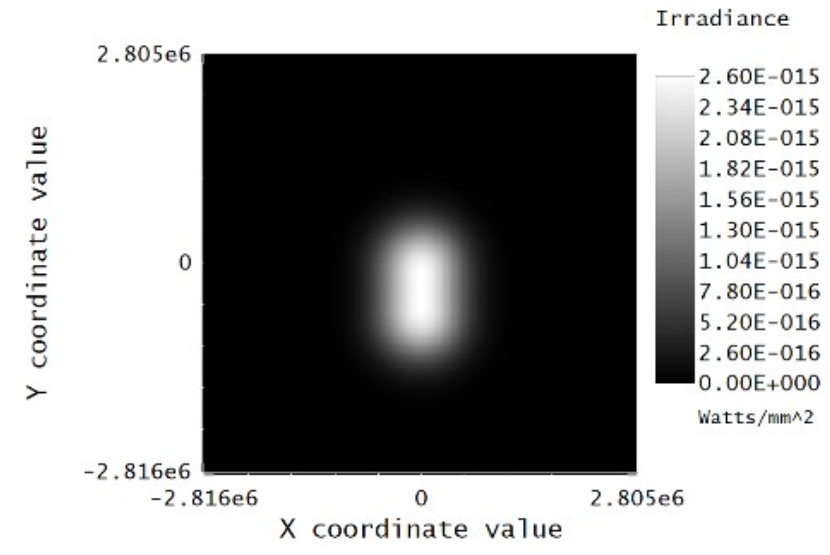

10a)

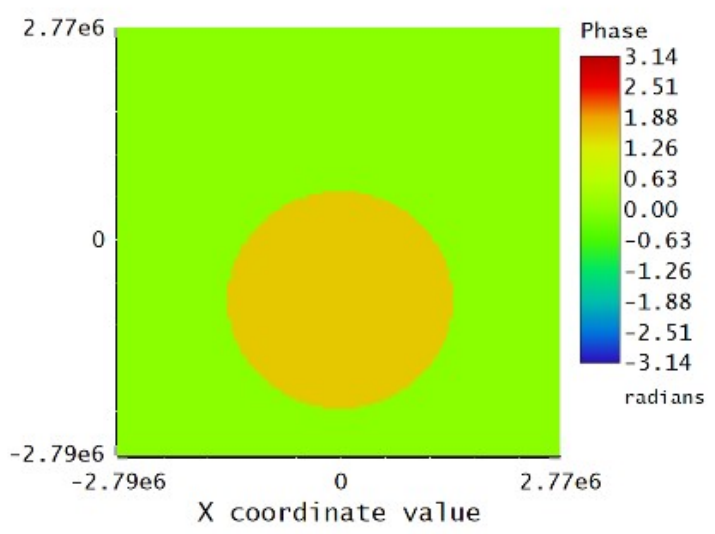

10b)

Figure 10: a) two overlapping incoherent laser beams that are pointed at $0^{\circ}$ and $0.075^{\circ}$ and which both have $2 \mathrm{~mW}$ of power, b) phase of the beam pointed at $0.075^{\circ}$.

Figure 11 shows the irradiance at $611 \mathrm{~km}$ for 5 laser beams that are pointed at $0^{\circ}$ and at $\pm 14^{\circ}$ in the $\mathrm{x}$ and $\mathrm{y}$ coordinate directions, and each has $2 \mathrm{~mW}$ of power. $\mathrm{AFOV} \Theta=28^{\circ}, \mathrm{FOV}$ (diameter) $304 \mathrm{~km}$ at $611 \mathrm{~km}$. As mentioned just after Fig. 1, the power of a beam can be increased by using a cluster of lasers. Figure 2 shows laser clusters.

Figure 12 shows the irradiance from 2 lasers in a cluster pointed at $0^{\circ}$, which is about double that from one laser, and the two beams overlap to form essentially one beam.

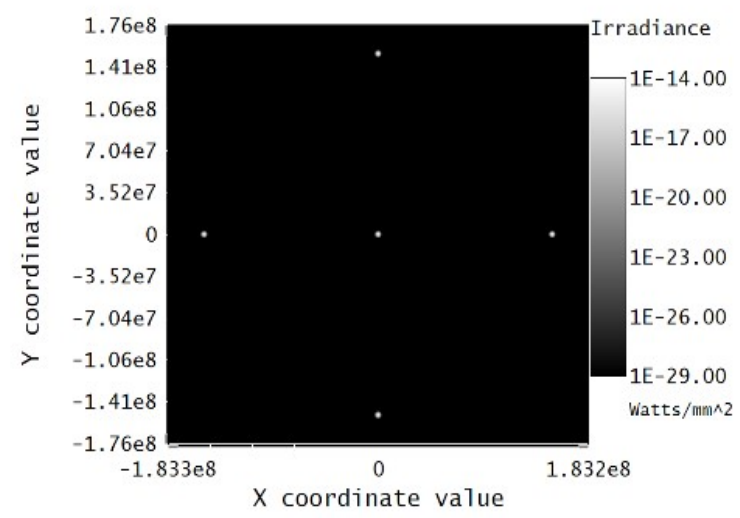

Figure 11: 5 laser beams

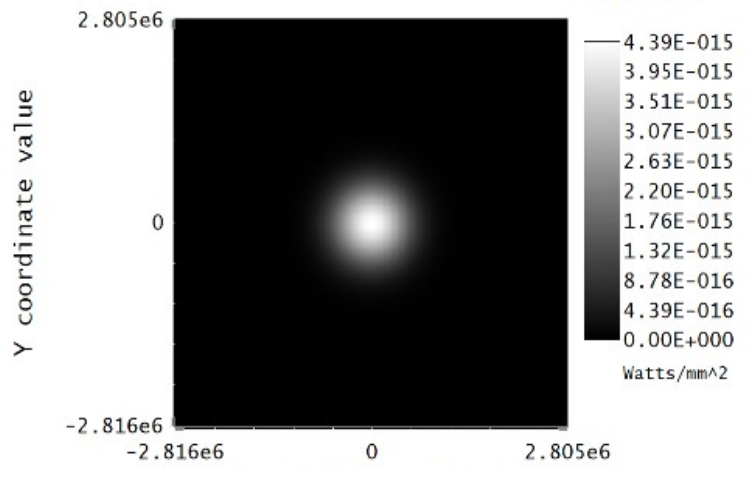

Figure 12: 2 lasers in a cluster

\section{LEO APPLICATION}

Preliminary analysis $\underline{2}$ indicates that this system can be incorporated into a small telescope to make the steering finer and consequently be applicable to transmissions between satellites in low-Earth orbit (LEO) and ground terminals. Figure 13 shows a lens design where a small telescope is placed in front of the lens/laser array system to reduce the angle $\beta$ of the beam propagation emitted from the lens/laser array system to the smaller angle $\alpha$ of the beam propagation emitted from the objective lens of the telescope. Without the telescope, the spots from two adjacent laser beams from a satellite in LEO may not overlap on the Earth, so there would be a loss of coverage of the beam at the ground terminal as the 
direction from the lens system to the ground terminal changed. The telescope design details depend on the design parameters of the lens/laser array system, the distance to the ground terminal, the size of the beam spot size, and the sensitivity of the ground receiver. The length 2 of the system can be reduced by using two or more telescope lens system in series, since the total magnification of the complete system is equal to the product of the separate magnifications. The magnification of the telescope $M=f_{o} / f_{e}$, where $f_{o}$ is the focal length of the objective lens and $f_{e}$ is the focal length of the eyepiece lens. Also, $M=\beta / \alpha$, so that the angle of the outgoing beam is reduced for finer aiming.

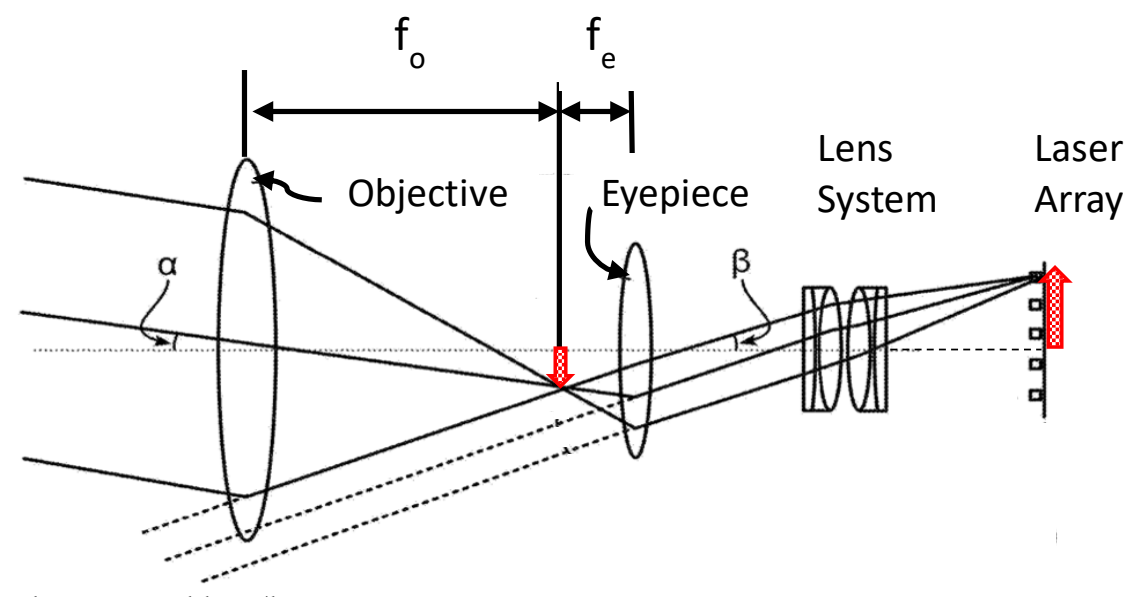

Figure 13: A telescope and lens/laser array system.

\subsection{Example of Telescope Lens System}

An application to the OCSD Program $\frac{10}{n}$ will be made to show how this new lens system can be used to augment a laser pointing system on a CubeSat that uses star trackers for body pointing. (It is noted ${ }^{10}$ that a secondary approach would be to use an uplink ground laser beacon and an onboard quad cell for closed-loop pointing.) The new lens system would provide a fine pointing capability to the OCSD lens system. With the resulting more accurate pointing, the power requirement would be substantially reduced and the resulting thermal load also reduced, thereby mitigating the current thermal load challenge ${ }^{10}$.

The OCSD mission utilized two CubeSats AeroCube-7B\&C. The divergences of the output beams for 7B and 7C were set conservatively to be $\sim 0.06^{\circ}$ and $0.15^{\circ} \mathrm{FWHM}$, respectively. In this section the results of computer simulations of the propagation of a laser beam for a LEO distance of $611 \mathrm{~km}$ will be shown. A divergence of $0.14^{\circ} \mathrm{FWHM}$ will be used in these simulations in order to obtain a spot size diameter at FWHM of $1600 \mathrm{~m}$ at the image plane. Then the results of computer simulations using the new lens system will be shown. The results will show that the power requirement of the beam can be reduced from $2 \mathrm{~W}$ to $90 \mathrm{~mW}$. These results are meant to only show a proof-of-concept, since the lenses used for both simulations were not designed for use in space communications.

\subsection{AeroCube-7C Propagation}

Figure 14 shows the irradiance and phase of the wave front at $611 \mathrm{~km}$ for the laser beam, which has a divergence of $0.14^{\circ} \mathrm{FWHM}$. The output power from the laser is $2 \mathrm{~W}$. The same lens from Section 3 was used, which is a wide-angle lens, double Gauss with $28^{\circ}$ field, as shown in Figs. 6 and 7. This lens is used for cameras and is used here just for simulating the beam propagation for $611 \mathrm{~km}$. The peak irradiance of the beam is $7.11 \mathrm{E}-13 \mathrm{~W} /(\mathrm{mm})^{2}$. The irradiance has a Gaussian profile, and the phase is essentially flat, so that this beam is diffraction limited. The units for the distances are in $\mathrm{mm}$ and the spot size is about $1600 \mathrm{~m}$. 


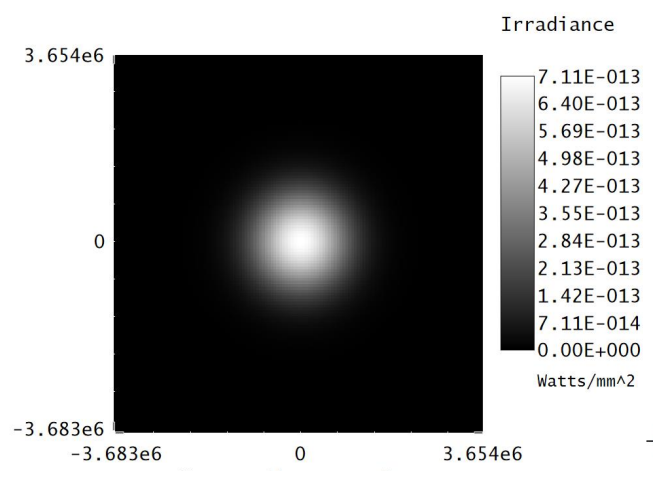

Figure 14: a) irradiance,

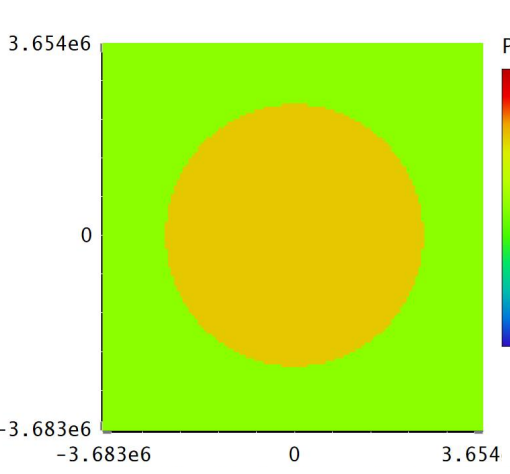

b) phase,

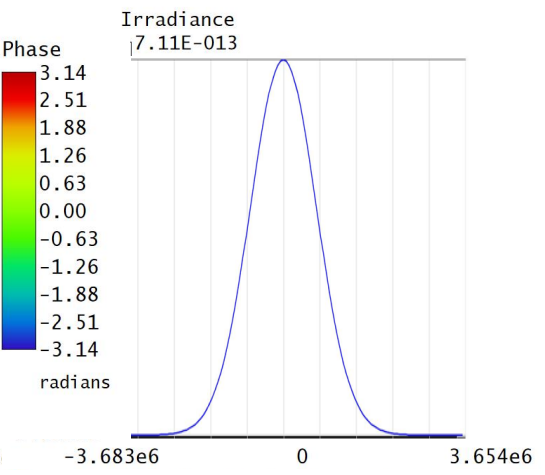

c) Y-coordinate crosssection of irradiance

\subsection{New Lens System Propagation}

A new lens design is shown in Fig. 15. It consists of three lens systems, each with four lens elements. The four lens elements consist of two doublets with the left doublet's elements in reverse order from that of the right doublet. This lens system is an example of a modern version of the symmetrical Plössl design, which is used as an eyepiece for telescopes. .11 The lens system on the right was the starting system in designing the new system. The Plössl lens is a simpler lens system than the double Gauss, which has six lens elements. The small middle lens system is a reduction of the right system by a factor of sixteen; so they form a miniature telescope with a magnification factor of sixteen. The left lens system converts the diverging beam from the left to a colinear beam for entry into the telescope system. It retains the radii of the right system and its focal length to minimize the angle from the focal plane of the first lens system from adjacent laser beams of the laser array to the vertex of the first surface of that lens system. In addition, there are microlenses ${ }^{12-14}$ (not shown) in front of each laser in the array to narrow the emerging beam and point it to the center of the lens systems stop, which is located between the first and second lens systems. The focal plane is where the middle lens focuses the beam. The distances from the last surface of the middle lens system to the focal plane and from the focal plane to the first surface of the right lens system indicate approximately fe, the focal length of the eyepiece lens and fo, the focal length of the objective lens respectively. The telescope's effect is to reduce the divergence of the beam and broaden the beam, both by the magnification of the telescope $M=f o / f e$, a factor of sixteen.

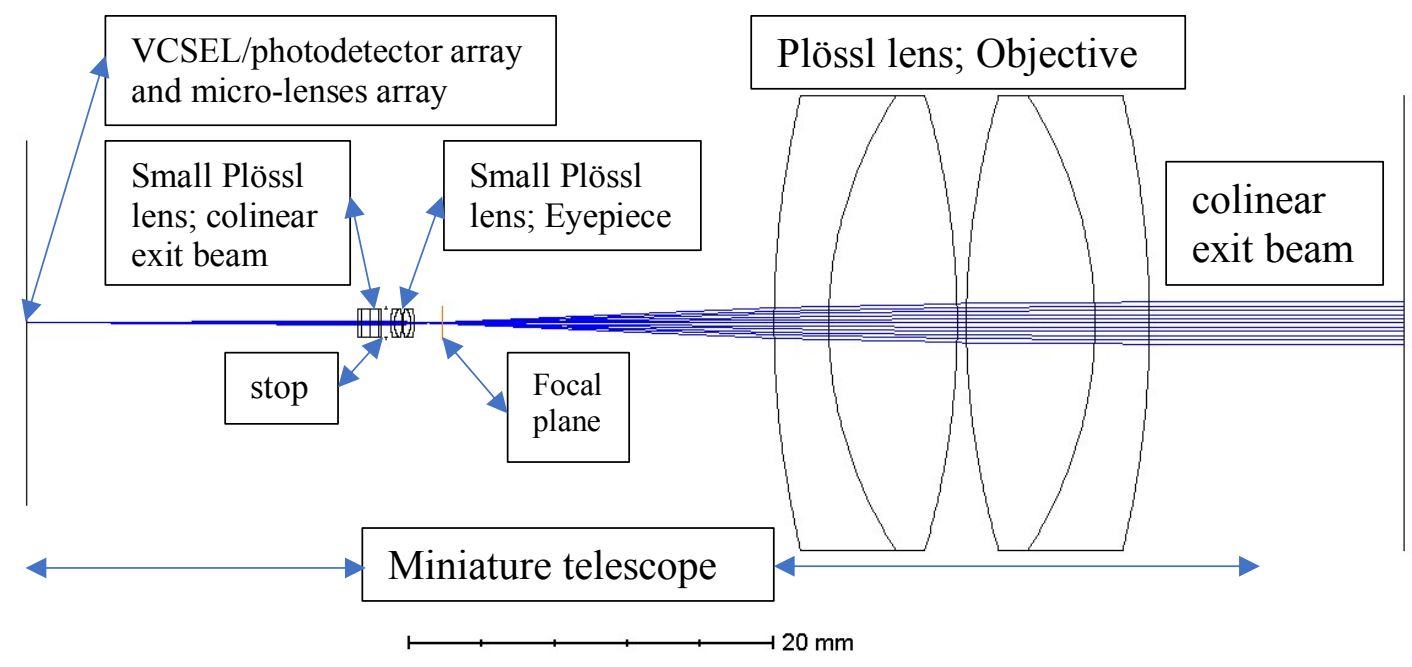

Figure 15: New lens system using Plössl lenses

A Plössl lens is used here just for simulating the beam propagation from the new lens system for a proof-of-concept demonstration. The length of the entire system is $48 \mathrm{~mm}$, from the location of the VCSEL/photodetector array on the 
left (vertical line) to the last lens surface on the right. The diameter of the lenses on the right is $25 \mathrm{~mm}$, which should be large enough to collect enough light for the photodetectors to detect the incoming beam. However, it may be possible to design a lens system that is much smaller. The Plössl lens was not designed for space communications, and the beam diameter at the last lens surface is $1.4 \mathrm{~mm}$ FWHM. (The beam shown in Fig. 15 is for illustrative purposes; the actual beam from the calculations is narrower.)

Figure 16 shows the irradiance and phase of the wave front at $611 \mathrm{~km}$ for the laser beam from the new lens system shown in Fig. 15. The laser beam has a divergence of $0.0174^{\circ}$ FWHM and the diameter of the spot size at the image plane is about $200 \mathrm{~m}$. The output power from the laser cluster is $30 \mathrm{~mW}$, and the peak irradiance of the beam is $7.15 \mathrm{E}-$ $13 \mathrm{~W} /(\mathrm{mm})^{2}$, which is almost the same as the $7.11 \mathrm{E}-13 \mathrm{~W} /(\mathrm{mm})^{2}$ for the $2 \mathrm{~W}$ beam with the divergence of $0.14^{\circ} \mathrm{FWHM}$. The irradiance has a Gaussian profile, and the phase is essentially flat, so that this beam is diffraction limited. The units for the distances are in $\mathrm{mm}$.

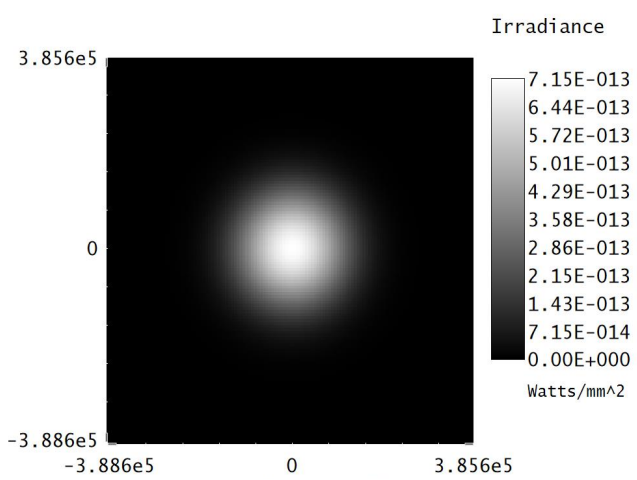

Figure 16: a) irradiance,

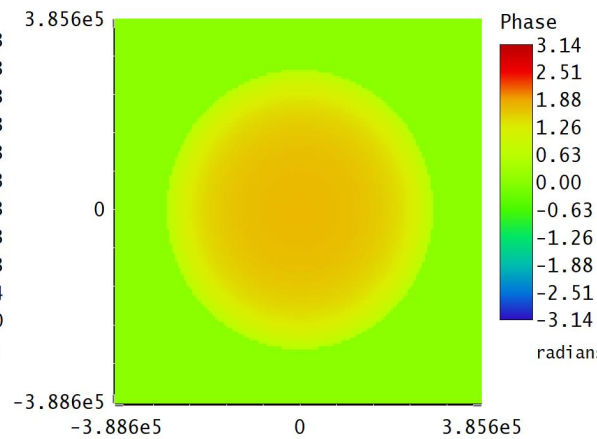

b) phase,

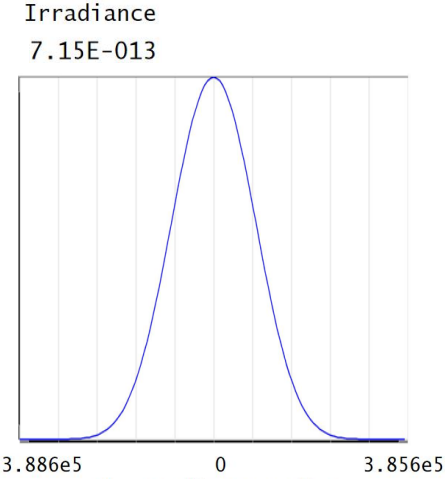

c) Y-coordinate cross- section of irradiance

\subsection{Multiple Beams Propagation}

Next the results of the simulation of two and then three incoherent beams simultaneously propagating will be shown. The resulting combined beam will form a plateau at the peak, so that changes in direction of that beam with respect to the receiver will not affect the intensity of the beam at the receiver, as long as the receiver is within the plateau area. This insensitivity is not possible if just one beam is transmitted.

The results in Fig. 16 are for a VCSEL or VCSEL cluster, as shown in Fig. 2, in the array at the focal plane of the first lens system whose center is located on the axis of the lens system, so the $\mathrm{x}$ and $\mathrm{y}$ coordinates of the center in the focal plane are $\mathrm{x}=\mathrm{y}=0$. Now results will be shown for $\mathrm{x}=0, \mathrm{y}=-87 \mu \mathrm{m}$. The pitch between the two lasers or laser clusters is $87 \mu \mathrm{m}$.

Figure 17 shows the irradiance and phase of the wave front at $611 \mathrm{~km}$ for the laser beam from the laser located at $\mathrm{x}=0$, $\mathrm{y}=-87 \mu \mathrm{m}$. Again, the output power from the laser cluster is $30 \mathrm{~mW}$ and now the peak irradiance of the beam is 7.14E$13 \mathrm{~W} /(\mathrm{mm})^{2}$. As for the beam from the laser located at $\mathrm{x}=0, \mathrm{y}=0 \mu \mathrm{m}$, the irradiance has a Gaussian profile, and the phase is essentially flat, so that this beam is diffraction limited. The center of the beam is about $200 \mathrm{~m}$ down from the center of the beam in Fig. 16. 


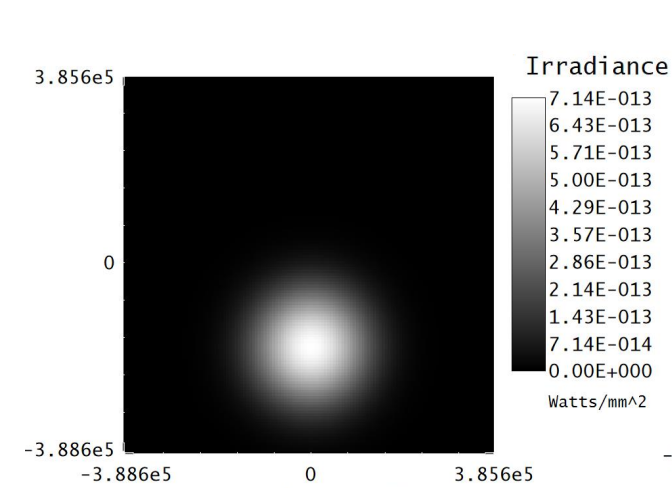

Figure 17: a) irradiance,

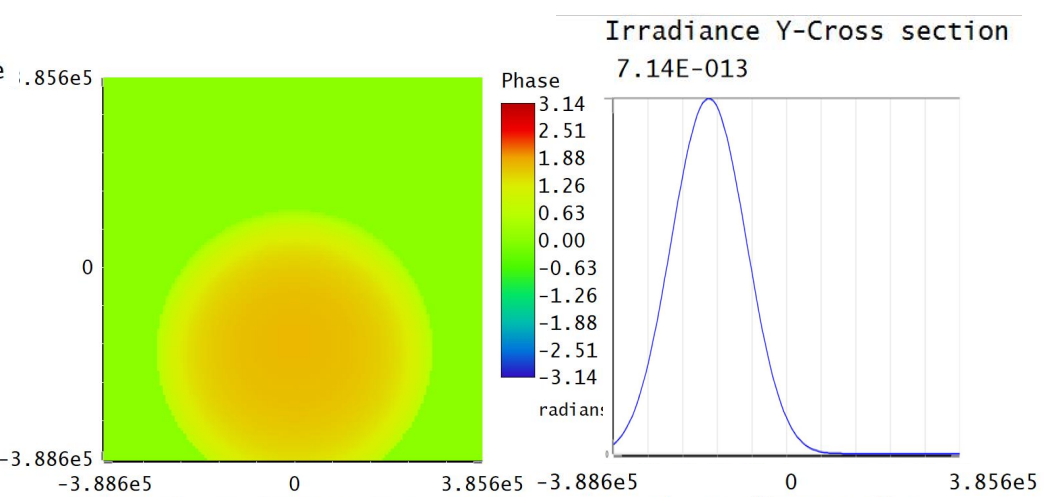

b) phase,

c) Y-coordinate cross- section of irradiance

Figure 18 shows the resulting beam when the two lasers are turned on together; the beam is the combination of the two incoherent beams shown in Figs. 16 and 17. There is a plateau region formed by the two laser beams. There is no phase plot since the beams are incoherent. The output power from the two laser clusters is $60 \mathrm{~mW}$.
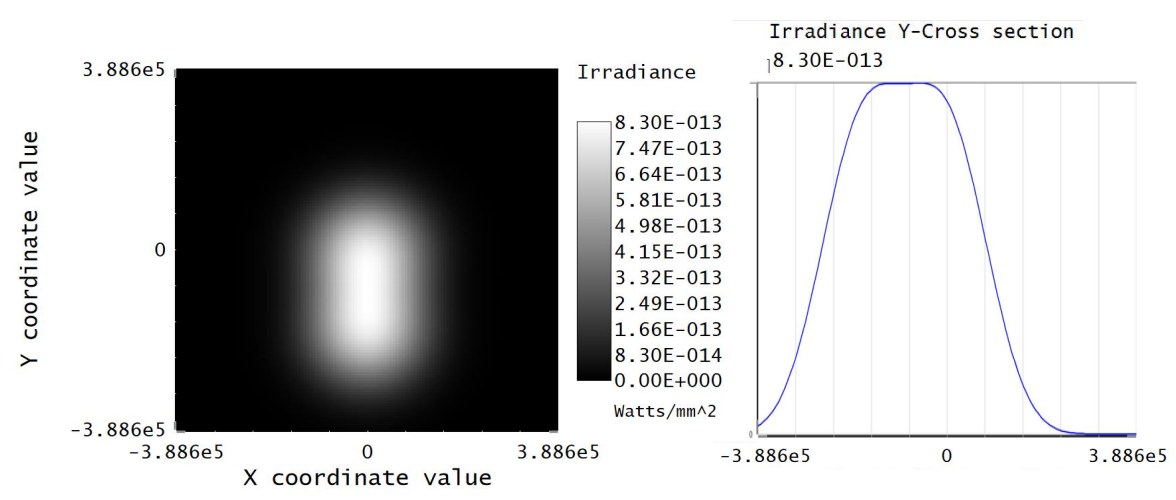

Figure 18: Two combined beams, a) irradiance,

b) Y-coordinate cross-section of irradiance.

In Fig. 19, results are shown for $\mathrm{x}=-75, \mathrm{y}=-44 \mu \mathrm{m}$. The pitch between the three lasers or laser clusters is $87 \mu \mathrm{m}$. As for the two-beam case, the irradiance for the third beam has a Gaussian profile, and the phase is essentially flat, so that this beam is diffraction limited. The center of the beam is equidistant from the centers of the beams in Figs. 16 and 17.

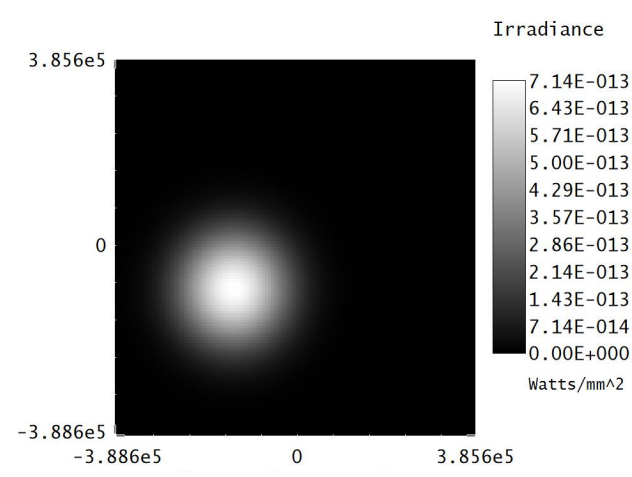

Figure 19: a) irradiance,

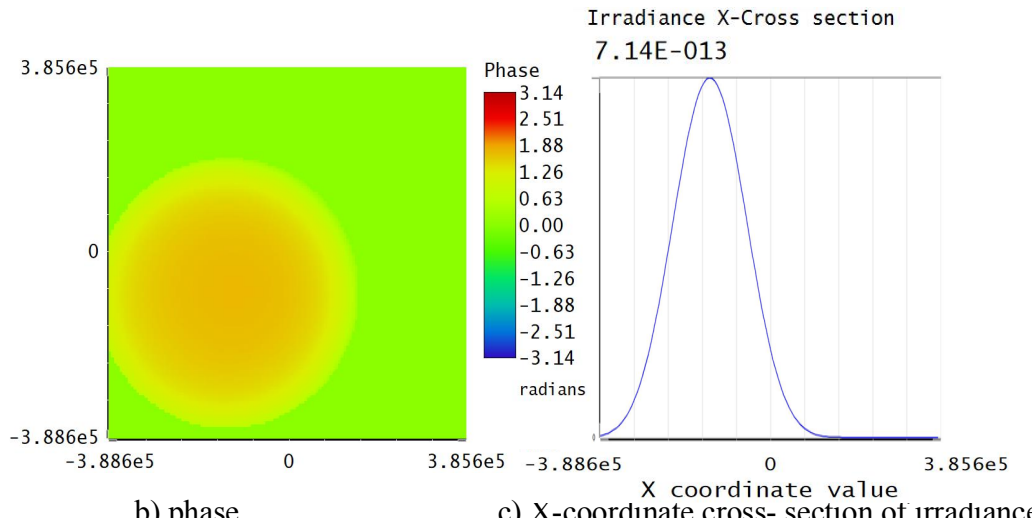

b) phase,

c) X-coordinate cross- section of irradiance

Figure 20 shows the resulting beam when the three lasers are turned on together; the beam is the combination of the three incoherent beams shown in Figs. 16, 17 and 19. They illuminate a triangular area. There is no phase plot since the beams are incoherent. In Fig. 20 b), the x- coordinate cross-section is taken at a y value half way between the centers of 
the two beams in Fig. 18, at which $y=-100 \mathrm{~m}$. The output power from the three laser clusters is $90 \mathrm{~mW}$. With three beams the pitch can be increased or the power of the lasers can be reduced and a plateau region will be produced.

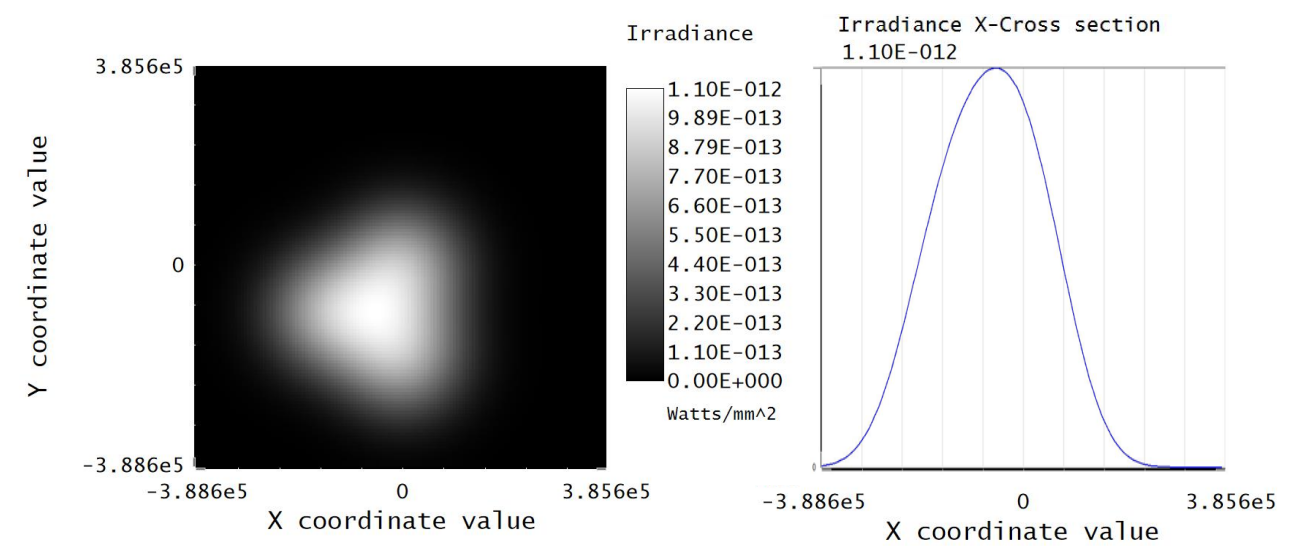

Figure 20: Three combined beams, a) irradiance,

b) X-coordinate cross-section of irradiance

\subsection{VCSEL/ Photodetector Array for Three Beam Transmission}

For the beam shown in Fig. 20 a), a different pattern of VCSEL clusters and photodetectors is preferable to the one shown in Fig. 2 for coupling the detection of the incoming signal beam with the transmission of the outgoing beam. Also, the shape of the photodetectors will be changed.

Figure 21 shows a candidate VCSEL/ Photodetector Array. In this array the detection of an incoming signal beam by a PIN would turn on the VCSEL clusters at the vertices of that element. As the signal beam changes direction to an adjacent PIN, the VECEL cluster not already on for it would be turned on and the corresponding one on the other PIN turned off.
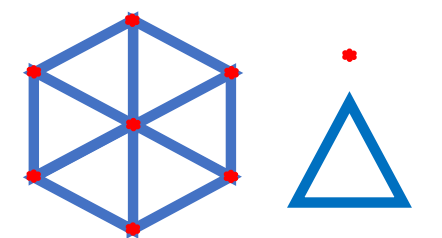

\section{VCSEL CLUSTER}

PIN

Fig. 21: Candidate VCSEL/ Photodetector Array

\subsection{Fine Pointing Capability}

Now a comparison will be made of the laser beam from one laser with an output power of $2 \mathrm{~W}$, as shown in Sec. 4.2 and of the laser beam from three laser clusters with an output power of $90 \mathrm{~mW}$. The spot size of the laser beam shown in Sec. 4.2 is $1600 \mathrm{~m}$ in diameter FWHM and the spot size of one beam in Sec. 4.3 is $200 \mathrm{~m}$. As shown in Fig. 18, a distance of $200 \mathrm{~m}$ between the centers of the spots assures a plateau region at the peak intensity. With three lasers, as shown in Fig. 20, a distance of $200 \mathrm{~m}$ between the centers of the spots produces a peak intensity greater than that shown in Sec. 4.2., so the distances between the three laser beam centers could be increased or the powers could be decreased. By increasing the number of PIN elements in Fig. 21 but keeping the pattern the same, so that there are 64 PIN elements and 42 VCSEL clusters,,$\frac{8}{12-14}$ the area covered by all the beams would cover the area covered by the beam shown in Fig. 14. Now the procedure explained in Sec. 2.1 could be applied. When a PIN detects an incoming signal beam, the three VCSEL clusters at its vertices would be turned on. A VCSEL cluster could consist of seven VECELs, each with a power of about $4 \mathrm{~mW}$. This fine pointing capability would augment the pointing system in AeroCube-7C. With more accurate pointing, the power requirement for the laser beam would be reduced and the resulting thermal load would be reduced. The fine pointing device is static and would maintain the avoidance of an optical gimbal or any internal beam steering hardware that has moving parts, as was done in AeroCube-7C..$^{10}$ The OCSD mission utilized two CubeSats AeroCube-7B\&C. The divergences of the output beams for 7B and 7C were set conservatively to be $\sim 0.06^{\circ}$ and $0.15^{\circ}$ 
FWHM, respectively. If the new fine pointing method were to be used to cover the area covered by AeroCube-7B, which is about 6.25 times smaller, then the power in each VCSEL cluster could be reduced to about $12 \mathrm{~mW}$ from 30 $\mathrm{mW}$ by reducing the area covered by each VCSEL cluster similarly. In a cluster of seven VCSELs, the power output in each VCSEL would be reduced from about $4 \mathrm{~mW}$ to $2 \mathrm{~mW}$. Alternatively, the number of PIN elements and VCSEL clusters could be reduced.

\section{RELATED TOPICS}

\subsection{Data Rate Transmission and Possible Power Levels for VCSEL Arrays}

VCSEL and VCSEL array devices can operate at data rates of $10 \mathrm{~Gb} / \mathrm{s}, 40 \mathrm{~Gb} / \mathrm{s}$, or higher $\frac{12-14}{\text {. However, there are recent }}$ developments $\frac{15}{15}$ to demonstrate a direct-to-Earth optical communication link from a CubeSat in low-Earth orbit at burst rates up to $200 \mathrm{Gbps}$. Similarly, there are recent developments ${ }^{16}$ in increasing data rates for VCSELs. From Ref. 16: "Actual standards and commercialized VCSEL are providing $25 \mathrm{~Gb} / \mathrm{s}$ data rates, but new solutions are expected to settle the next device generation enabling $100 \mathrm{~Gb} / \mathrm{s} . "$

The devices studied in Sec. 4 employed VCSELs with output powers around $4 \mathrm{~mW}$ in clusters of seven elements with output power of about $30 \mathrm{~mW}$. However there are VCSELs and VCSEL arrays with much higher powers, with output peak powers ${ }^{12-14}$ of $160 \mathrm{~W}$ and pulse widths of $7.2 \mathrm{~ns}$ (FWHM), and others $\frac{17}{17}$ with output powers of almost $10 \mathrm{~kW}$. So, laser beams for space optical communications that require more power, such as a few watts, can be obtained with VCSEL clusters with more elements or with VCSELs with larger apertures.

\subsection{Optical Multiple Access and Communication Among a Constellation of Satellites}

There are recent developments ${ }^{18}$ for "an inter-satellite omnidirectional optical communicator (ISOC) that will enable up to $1 \mathrm{Gbps}$ data rates over a distance up to $200 \mathrm{~km}$ in free space. Key features of the ISOC include full sky coverage and its ability to maintain multiple links simultaneously. The ISOC outer frame is a truncated icosahedron small enough to deploy out of a CubeSat. The frame contains photodetector receivers and gimbal-less scanning MEMS mirrors for transmit beam steering. There are miniature transmitter housings ("telescopes") that include a single mode laser diode and a MEMS mirror. The frame's vertices feature fast photodetectors for reception and direction finding." To operate MEMS mirrors, voltages of around $\frac{19}{10} \mathrm{~V}$ to around $^{20} 100 \mathrm{~V}$ are used.

A possible alternative would be to use the method presented in Sec. 4, a lens system/VCSEL-Photodetector Array system, together with two steering MEMS mirrors that require less voltage ${ }^{21}$, around hundreds of $\mathrm{mV}$. The mirrors would provide the pointing over the larger angles and the new system would provide the fine pointing on a nanosecond time scale. Also, the random angular disturbance caused by satellite vibration $\frac{18}{\underline{ }}$ would be taken care of electronically by the VCSEL-Photodetector Array, also on a nanosecond time scale.

\subsection{Extension to Longer, Planetary Distances}

With further design studies, it may be possible to extend this method to longer, planetary distances ${ }^{22}$. The size of the lens system would probably need to be scaled up and the power of the laser clusters would have to be increased. At such large distances it is crucial to "isolate the optical transceiver assembly from base platform vibrational disturbances by an isolation pointing assembly", since any minor shift in direction would cause the laser beam to miss the receiver.

However, with the use of a lens system/VCSEL-Photodetector Array system to provide fine pointing, the effects of vibrational disturbances would be mitigated on a nanosecond time scale.

\section{SUMMARY}

A new method has been described for optical data transmissions from satellites using laser arrays for laser beam pointing. It combines a lens system and a VCSEL/Photodetector Array, both mature technologies, in a novel way. The concept is described and some features of the VCSEL/Photodetector Array are explained. This static system for beam pointing can replace current architectures which use dynamical systems (i.e., moving parts such as fast-steering mirrors (FSM), and/or gimbals) to point the laser, and which may use vibration isolation platforms. Preliminary results of 
computer simulations of the new system show diffraction-limited beam propagation. These simulations serve to show a proof-of-concept of the method to propagate diffraction limited laser beams in different directions from lasers in the focal (object) plane of the lens system for LEO distances. In Sec. 4, a possible application to the OCSD Program was described. Possible additional applications are to planetary distances (deep space optical communications, DSOC), to optical multiple access, to communication between a constellation of close satellites, and to satellites that use modulating retro-reflectors.

\section{REFERENCES}

[1] U.S. Patent No. 9,774,395, Space Optical Communications Using Laser Beams, September 26, 2017. http://patft.uspto.gov/netacgi/nph-Parser?Sect1 $=$ PTO2\&Sect2=HITOFF \&p=1\&u=\%2Fnetahtml $\% 2$ FPTO $\% 2$ Fsearchbool.html\&r=2\&f=G\&l=50\&co1=AND\&d=PTXT\&s1=goorjian.INNM.\&OS=IN/goorjian\&RS=IN/goorjian [2] U.S. Patent No. 9,954,613, Methods and Devices for Space Optical Communications Using Laser Beams, April 24, 2018.

http://patft.uspto.gov/netacgi/nph-Parser?Sectl=PTO2\&Sect2=HITOFF \&p=1\&u=\%2Fnetahtml\%2FPTO $\% 2$ Fsearchbool.html\&r=1\&f=G\&l=50\&co1=AND\&d=PTXT\&s1=goorjian.INNM.\&OS=IN/goorjian\&RS=IN/goorjian https://ntrs.nasa.gov/search.jsp? $\mathrm{R}=20180002686 \&$ hterms $=$ goorjian\&qs $=\mathrm{N} \% 3 \mathrm{D} 0 \% 26 \mathrm{Ntk} \% 3 \mathrm{DAll} \% 26 \mathrm{Ntx} \% 3 \mathrm{Dmode} \% 2$ Bmatchallany\%26Ntt\%3Dgoorjian

Goorjian, P. M., "A New Laser Beam Pointing Method Using Laser Arrays”, NASA/TM-2018-58815, November 2018. [3] Market Assessment for NASA Ames Research Center, Technology Partnerships Division, October 14, 2015, NTR Number: ARC-17122-1B, "Space Optical Communications using a New Laser Beam Pointing Technology for Optical Data Transmissions from Spacecraft".

[4] Keck Institute for Space Studies (KISS) workshop "Optical Communication on SmallSats - Enabling the Next-Era in Space Science", July 11-14, 2016 and February 2-9, 2017 at CIT, Pasadena, CA.

[5] Alberto, A. G. et al., "Modulating Retro-Reflectors: Technology, Link Budgets and Applications", 63rd

International Astronautical Congress, Naples, Italy, paper IAC-12, B4,6B,11 (2012).

[6] OpticStudio, Zemax. LLC; https://www.zemax.com

[7] Edmund Optics https://www.edmundoptics.com

[8] Choquette, K, D. et al., "2-Dimensional Integrated VCSEL and PiN Photodetector

Arrays for a Bidirectional Optical Links", 2007 IEEE Aerospace Conference, IEEEAC paper \#1042, https://ieeexplore.ieee.org/document/4161431,

Giannopoulos, A. V., Kasten, A. M., Long, C. M., Chen, C., and Choquette, K, D. "Two-dimensional integration of a vertical-cavity surface-emitting laser and photodetectors for position sensing," Applied Optics, Vol. 47, No. 25,

September 2008.

https://www.osapublishing.org/ao/abstract.cfm?uri=ao-47-25-4555

[9] http://www.photonics.philips.com/

[10] T.S. Rose et al., todd.s.rose@aero.org, 2018, “Optical Communications Downlink from a 1.5U CubeSat: OCSD Program", Proceedings of the 32 $2^{\text {nd }}$ AIAA/USU Conference on Small Satellites, Session 11: Assuring the Space

Ecosystem I, SSC18-XI-10, https://digitalcommons.usu.edu/smallsat/2018/all2018/485/

[11] Astronomical Optics, Part 1: Basic Optics http://www.handprint.com/ASTRO/ae1.html

[12] Carson, Richard F. et al., TriLumina Corp. "Compact VCSEL-based laser array communications systems for improved data performance in satellites", Proc. of SPIE Vol. 9226, 92260H (2014).

https://www.spiedigitallibrary.org/conference-proceedings-of-spie/9226/92260H/Compact-VCSEL-based-laser-arraycommunications-systems-for-improved-data/10.1117/12.2061786.full? SSO=1

[13] Warren, Mial E. et al., TriLumina Corp. "High-speed and scalable high-power

VCSEL arrays and their applications", Proc. of SPIE Vol. 9381, 93810C (2015).

https://www.spiedigitallibrary.org/conference-proceedings-of-spie/9381/93810C/High-speed-and-scalable-high-powerVCSEL-arrays-and-their/10.1117/12.2080235.full

[14] Carson, Richard F. et al., TriLumina Corp. "Progress in High-Power, High-Speed VCSEL Arrays", Proc. of SPIE Vol. 9766, 97660B (2016).

https://www.spiedigitallibrary.org/conference-proceedings-of-spie/10122/1012205/Progress-in-optimization-of-highpower-high-speed-VCSEL-arrays/10.1117/12.2253142.full

[15] Robinson, B. S. et al., "TeraByte InfraRed Delivery (TBIRD): A Demonstration of Large- 
Volume Direct-to-Earth Data Transfer from Low-Earth Orbit”, Proc. of SPIE Vol. 10524, 105240V (2018). https://www.spiedigitallibrary.org/conference-proceedings-of-spie/10524/105240V/TeraByte-InfraRed-DeliveryTBIRD--a-demonstration-of-large-volume/10.1117/12.2295023.full?SSO=1

[16] Marigo-Lombart, L. et al., "Integration of Electro-Absorption Modulator in a Vertical-Cavity Surface-Emitting Laser", Proc. of SPIE Vol. 10552, 105520L (2018)

https://www.spiedigitallibrary.org/conference-proceedings-of-spie/10552/105520L/Integration-of-electro-absorptionmodulator-in-a-vertical-cavity-surface/10.1117/12.2287465.full

[17] Moench, Holger. et al., "High power VCSEL systems and applications", Proc. of SPIE Vol. 9348, $93480 \mathrm{~W}$ (2015) http://www.photonics.philips.com/pdf/High\%20power\%20VCSEL\%20systems\%20and\%20applications.PDF

[18] Velazco, Jose E. et al., "High data rate inter-satellite Omnidirectional Optical Communicator", Proceedings of the $32^{\text {nd }}$ AIAA/USU Conference on Small Satellites, Session 1: Advanced Concepts I: SSC18-WKI-02, https://digitalcommons.usu.edu/cgi/viewcontent.cgi? article=4231\&context=smallsat https://digitalcommons.usu.edu/cgi/viewcontent.cgi?filename $=0$ \&article $=4231$ \&context $=$ smallsat\&type $=$ additional Zaman, Imam Uz et al., "Design Tradeoffs and Challenges of Omnidirectional Optical Antenna for High Speed, Long Range Inter CubeSat Data Communication", Proceedings of the $32^{\text {nd }}$ AIAA/USU Conference on Small Satellites, Session 2: Delivering Mission Success: SSC18-WKII-06

https://digitalcommons.usu.edu/cgi/viewcontent.cgi?article $=4241 \&$ context $=$ smallsat https://digitalcommons.usu.edu/cgi/viewcontent.cgi?filename $=0 \&$ article $=4241 \&$ context $=$ smallsat\&type $=$ additional [19] Optotune.com: 2D Beam Steering https://www.optotune.com/products/beam-steering

[20] Mirrorcle Technologies, Inc, https://www.mirrorcletech.com/wp/products/mems-mirrors/ https://mirrorcletech.com/pdf/Mirrorcle\%20Technologies\%20MEMS\%20Mirrors\%20-\%20Technical\%20Overview.pdf [21] Ye, Liangchen et al., "Large-Aperture kHz Operating Frequency Ti-alloy Based Optical Micro Scanning Mirror for LiDAR Application", https://www.mdpi.com/2072-666X/8/4/120, https://www.mdpi.com/2072-666X/8/4/120/htm [22] Biswas, Abhijit et al., "Deep Space Optical Communications", Proc. of SPIE Vol. 10524, 105240U (2018 ) https://www.spiedigitallibrary.org/Search/download?downloadType=Proceedings\%20Article\&DOI=10.1117\%2F12.229 $\underline{6426}$ 